\begin{tabular}{|c|l|}
\hline Title & $\begin{array}{l}\text { Hexagonal ferromagnetic MnA s nanocluster formation on Gal nA s InP (111)B layers by metal-organic vapor phase } \\
\text { epitaxy }\end{array}$ \\
\hline Author(s) & Hara, Shinjiroh; Fukui, Takashi \\
\hline Citation & $\begin{array}{l}\text { A pplied Physics Letters, 89(11), 113111 } \\
\text { https://doi.org/10.1063/2349309 }\end{array}$ \\
\hline Issue Date & 2006-09 \\
\hline Doc URL & http://hdl.handle.net/2115/14665 \\
\hline Rights & Copyright $\odot 2006$ A merican Institute of Physics \\
\hline Type & article \\
\hline File Information & APL 2006-89.pdf \\
\hline
\end{tabular}

Instructions for use 


\title{
Hexagonal ferromagnetic MnAs nanocluster formation on GalnAs/InP (111)B layers by metal-organic vapor phase epitaxy
}

\author{
Shinjiroh Hara ${ }^{\text {a) }}$ and Takashi Fukui \\ Research Center for Integrated Quantum Electronics (RCIQE), Hokkaido University, North 14 West 9 , \\ Sapporo 060-0814, Japan and Graduate School of Information Science and Technology, Hokkaido University, \\ North 13 West 8, Sapporo 060-8628, Japan
}

(Received 5 May 2006; accepted 18 July 2006; published online 12 September 2006)

\begin{abstract}
The authors report the self-assembly of hexagonal MnAs nanoclusters on GaInAs (111)B surfaces by metal-organic vapor phase epitaxy. The ferromagnetic behavior of the nanoclusters dominates the magnetic response of the samples when magnetic fields are applied in a direction parallel to the wafer plane. For the magnetic fields applied in a direction perpendicular to the plane, diamagnetic characteristics are dominant. The results indicate that the $c$ axis of the nanoclusters is perpendicular to the plane, and that their $a$ axis is in plane. They are consistent with the results of crystallographic analysis, where the nanoclusters' $c$ axis is shown to be along a GaInAs [-1-1-1] direction. (C) 2006 American Institute of Physics. [DOI: 10.1063/1.2349309]
\end{abstract}

Ferromagnetic materials hybridized in III-V compound semiconductors (FM III-V hybrids) and III-V compoundbased diluted magnetic semiconductors (III-V DMSs) are promising for the realization of nanospintronic devices using not only the charge but the spin of carriers. In MnAs/GaAs, GaMnAs, and InMnAs materials systems where extensive research efforts have been carried out, magnetic thin films have been grown on GaAs layers by molecular beam epitaxy at an extremely low growth temperature (LT-MBE), ${ }^{1-3}$ implantation of magnetic ions into semiconductors, ${ }^{4}$ and metalorganic vapor phase epitaxy (MOVPE). ${ }^{5,6}$ We have pursued FM III-V hybrids, in particular, using MnAs nanoclusters (NCs) embedded in GaInAs/InP (001) layers grown by MOVPE. $^{7,8}$ InP-related materials are more suitable for the fabrication of key devices in optical communication systems for 1.3 and $1.55 \mu \mathrm{m}$ wavelength bands. Recently, LT-MBE of III-V DMS, e.g., GaInMnAs, on InP (001) wafers has been reported, ${ }^{9,10}$ and waveguide-type optical isolators using the magneto-optical effects of MnAs NCs have been proposed. ${ }^{11}$ Ferromagnetic MnAs thin films, in addition, serve as an electrical spin injection source into semiconductors. $^{12}$

For the growth of hexagonal NiAs-type MnAs layers, $\{111\}$ orientations of zinc-blende (ZB)-type materials are promising because of the similarity of the crystallographic structures. MnAs "thin films" have been grown not only on GaAs (111)B (Refs. 13-18) but on Si (111) (Ref. 19) surfaces by LT-MBE. Another potential advantage using the $\{111\}$ orientations is the catalyst-free formation of onedimensional (1D) semiconductor nanowires (NWs) using selective-area MOVPE as promising building blocks for future nanophotonics and electronics. ${ }^{20,21}$ The hybridization of ferromagnetic NCs into 1D semiconductor NWs possibly leads to the understanding of physical properties in 1D spinpolarized electronic systems and the realization of 1D nanospintronic devices in the next generation. We believe that the MOVPE techniques in the present work are one of the most powerful methods to meet this future goal. In this letter, we

\footnotetext{
a) Author to whom correspondence should be addressed; electronic mail:
} hara@ rciqe.hokudai.ac.jp demonstrate the self-assembly of ferromagnetic MnAs NCs on planar GaInAs (111)B surfaces by MOVPE. This letter describes the results of fundamental crystallographic and magnetic characterizations for the NCs.

$\left(\mathrm{CH}_{3}\right)_{3} \mathrm{Ga},\left(\mathrm{CH}_{3}\right)_{3} \mathrm{In}, t-\mathrm{C}_{4} \mathrm{H}_{9} \mathrm{PH}_{2}$, and $20 \%-\mathrm{AsH}_{3}$ diluted in $\mathrm{H}_{2}$ were used as group III and $\mathrm{V}$ source materials. $\left(\mathrm{CH}_{3} \mathrm{C}_{5} \mathrm{H}_{4}\right)_{2} \mathrm{Mn}$ was chosen as a $\mathrm{Mn}$ organometallic precursor. $^{8}$ After the growth of GaInAs layers on InP buffer layers, $\left(\mathrm{CH}_{3} \mathrm{C}_{5} \mathrm{H}_{4}\right)_{2} \mathrm{Mn}$ was introduced to the MOVPE reactor with $\mathrm{AsH}_{3}$. All the layers were grown on diamagnetic InP (111)B wafers, and, as a reference, InP (001) substrates were used at the same growth runs. The growth temperature $T_{g}$ and the $\mathrm{V} / \mathrm{Mn}$ ratio were $600{ }^{\circ} \mathrm{C}$ and 375 , respectively. Here, the supply gas ratio between the partial pressures of a group $\mathrm{V}$ source, $p\left[\mathrm{AsH}_{3}\right]$, and a manganese precursor, $p\left[\left(\mathrm{CH}_{3} \mathrm{C}_{5} \mathrm{H}_{4}\right)_{2} \mathrm{Mn}\right]$, is defined as $\mathrm{V} / \mathrm{Mn}$ $=p\left[\mathrm{AsH}_{3}\right] / p\left[\left(\mathrm{CH}_{3} \mathrm{C}_{5} \mathrm{H}_{4}\right)_{2} \mathrm{Mn}\right]$ and is referred to as a "V/Mn ratio." Surface morphologies of the samples were observed by atomic force microscopy (AFM). Cross-sectional lattice images and electron beam diffraction (ED) patterns were observed to investigate the crystallographic structures of NCs by transmission electron microscopy (TEM), and energy dispersive $x$-ray (EDX) spectroscopy was carried out for the compositional analysis. Vibrating sample magnetometer was used to characterize magnetic properties of NCs.

Initially, we revealed by AFM that GaInAs layers grown on InP buffer layers had no NCs on the surfaces. A flat GaInAs surface with steps and large terraces was formed. We find that NCs are formed on GaInAs (111)B and (001) surfaces after the MnAs growth. As shown in Fig. 1, hexagonal NCs with well-defined sidewall crystal facets were formed on the (111)B surfaces. Such facets are attributable to some low and high index crystal planes of a hexagonal structure. Typical hexagonal NCs measured about $330 \mathrm{~nm}$ in diameter and $6-20 \mathrm{~nm}$ in height. The lateral size of the NCs was increased with increasing $p\left[\left(\mathrm{CH}_{3} \mathrm{C}_{5} \mathrm{H}_{4}\right)_{2} \mathrm{Mn}\right]$, whereas almost no change was observed with respect to the NC heights. The observed hexagonal NCs on the (111)B surfaces were mostly elongated toward a $[-110]$ direction. On the $(001)$ surfaces, on the other hand, rectangular NCs elongated toward a [110] direction were formed (not shown). Figure 2(a) 


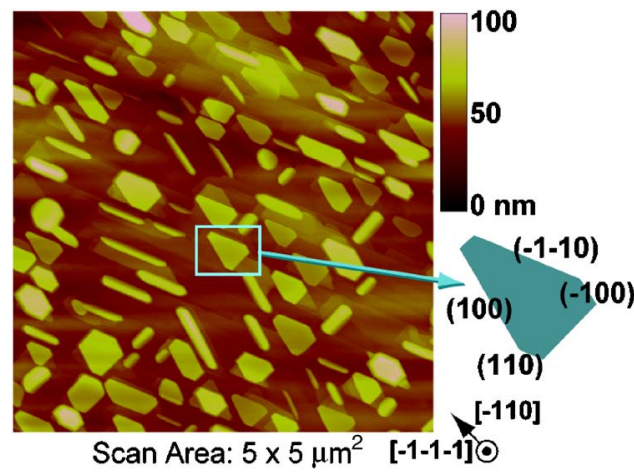

FIG. 1. (Color online) Surface morphology of hexagonal MnAs NCs grown on GaInAs (111)B surfaces.

shows a cross-sectional TEM image of the MnAs NC grown on GaInAs/InP (111)B layers. The observed NC measured about $250 \mathrm{~nm}$ wide and $23 \mathrm{~nm}$ high. The solid compositions of the hexagonal NCs examined by EDX spectroscopy are summarized in Table I. The analyzed regions are represented by the open circles 1 and 2 in Fig. 2(a). The NC on the
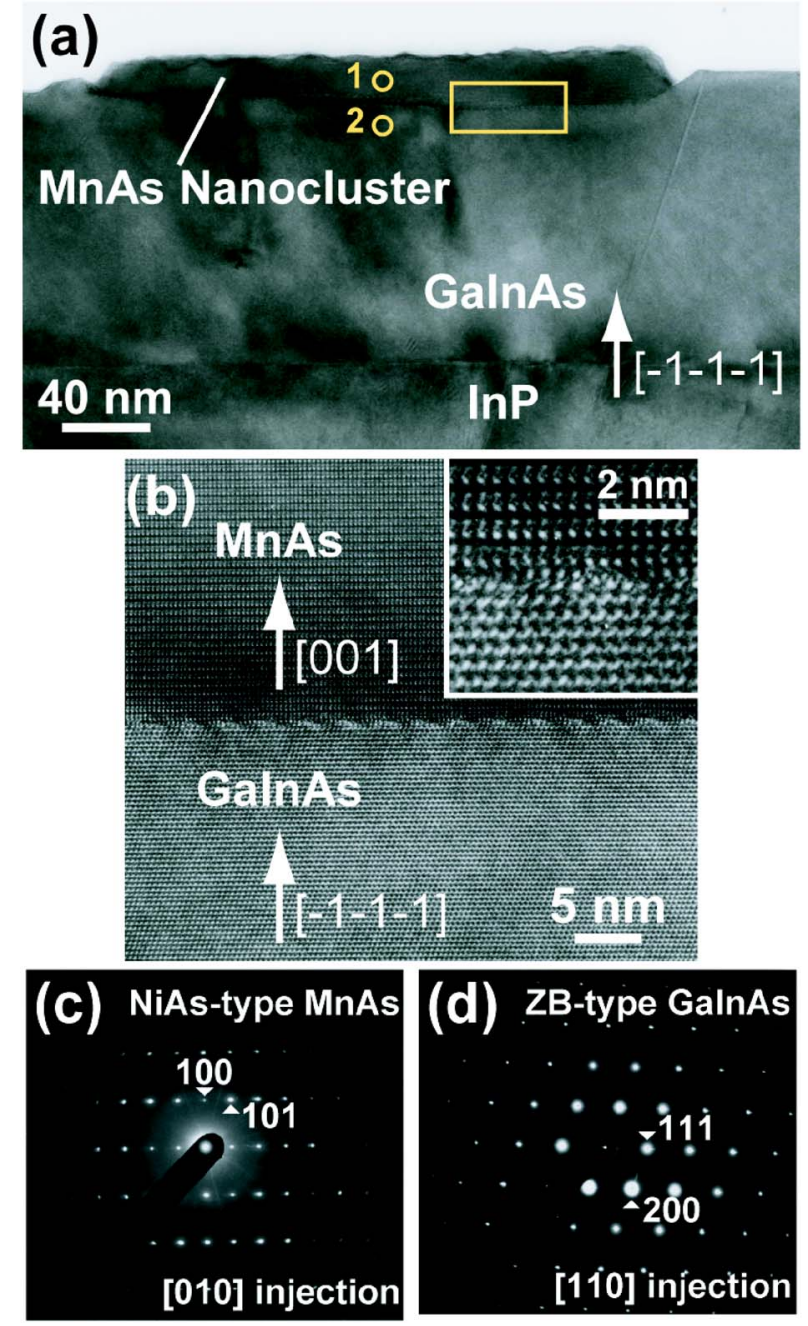

FIG. 2. (Color online) (a) Cross-sectional TEM image of a MnAs NC. The regions represented by open circles 1 and 2 are for the EDX analysis (Table I). (b) Lattice images of a MnAs NC and GaInAs layers for the area represented by a rectangular marker in (a). Inset is a highly magnified image around the interface. ED patterns from (c) a MnAs NC and (d) GaInAs layers indicate that a [001] direction of a NiAs-type MnAs NC is parallel to

a [-1-1-1] direction of ZB-type GaInAs layers. $\quad$ tion, as shown in Fig. 2 . It has been reported that the LT-
Downloaded 12 Sep 2006 to 133.87 .129 .68 . Redistribution subject to AlP license or copyright, see http://apl.aip.org/apl/copyright.jsp
TABLE I. Solid compositions of a MnAs NC and GaInAs layers analyzed by EDX spectroscopy. The focused spot diameter of electron beam for the analysis was about $1 \mathrm{~nm}$.

\begin{tabular}{ccccc}
\hline \hline & \multicolumn{4}{c}{ Compositions (at. \%) } \\
\cline { 2 - 5 } $\begin{array}{c}\text { Analyzed regions } \\
\text { open circles in Fig. 2(a) }\end{array}$ & Ga & In & As & Mn \\
\hline $\begin{array}{c}1 \\
\text { (MnAs nanocluster) } \\
2\end{array}$ & 0.6 & 1.0 & 52.1 & 46.3 \\
(GaInAs layers) & 19.6 & 30.4 & 49.4 & 0.6 \\
\hline \hline
\end{tabular}

GaInAs surfaces consisted of a mixture of Mn and As atoms, whereas Mn concentrations in the underlying GaInAs layers were almost negligible. Figure 2(b) shows cross-sectional lattice images of the MnAs NC and the GaInAs layers. It has been reported that some small pinholes ${ }^{16}$ and dislocations with a screw component ${ }^{17}$ have been observed in MnAs thin films grown by LT-MBE on GaAs (111)B surfaces, although the atomically flat terraces have been evolved between the steps on the MnAs surfaces. We find, however, that no dislocations are observed in the MnAs NC, and that it has epitaxially grown on GaInAs/InP (111)B layers. The close observations, in addition, demonstrated that an atomically flat interface between MnAs and GaInAs layers was formed, as shown in the inset of Fig. 2(b). These results are possibly because nanometer sized clusters with a strain, which is caused by a lattice mismatch between MnAs and GaInAs, have been formed, and because the growth processes in the MOVPE are strongly dominated by the kinetics of surface reactions, which possibly lead to morphological defect-free epitaxial layers. We conclude from ED patterns of Figs. 2(c) and 2(d) that the MnAs NCs have hexagonal NiAs-type crystallographic structures (MnAs: $a=0.372 \mathrm{~nm}$ and $c$ $=0.571 \mathrm{~nm}$ ), and that the $c$ axis of the NCs is parallel to the [-1-1-1] directions of ZB-type GaInAs layers.

Subsequently, we have observed strong ferromagnetic behavior and magnetic anisotropy in the MnAs NCs on GaInAs (111)B surfaces. Figures 3(a) and 3(b) show the detailed angle dependences of the magnetizations normalized by the ones under the external magnetic fields $\mathbf{H}$ applied at $\theta$ and $\phi=0^{\circ}$, i.e., $\mathbf{H} \|$ wafer plane. The measurements were carried out under the conditions of $\mathbf{H}=1.5 \mathrm{kOe}$ at room temperature. $M-H$ curves observed at $\theta=0^{\circ}$ [Fig. 3(a)] and $\phi$ $=90^{\circ}$ [Fig. 3(b)] are shown in Figs. 3(c) and 3(d), respectively. In the case of "in-plane rotation" in Fig. 3(a), in which $\mathbf{H}$ was always applied in a direction parallel to the InP (111)B wafer planes, clear hysteresis loops were observed, and it showed no significant dependences on the rotation angles. In the case of "out-of-plane rotation" in Fig. 3(b), on the other hand, diamagnetic characteristics mainly originated from GaInAs/InP were observed when the applied $\mathbf{H}$ was in a direction perpendicular to the wafer planes, although clear hysteresis loops were observed for other rotation angles. The magnetic characterizations, therefore, demonstrate that a magnetic hard axis of the hexagonal MnAs NCs, i.e., the $c$ axis, is perpendicular to the wafer planes, and that their magnetic easy axis, i.e., the $a$ axis, is in plane. These magnetic properties are consistent with the results of cross-sectional TEM observation, in which a [001] direction ( $c$ axis) of the NCs is shown to be parallel to a GaInAs $[-1-1-1]$ direction, as shown in Fig. 2. It has been reported that the LTAIP license or copyright, see http://apl.aip.org/apl/copyright.jsp 

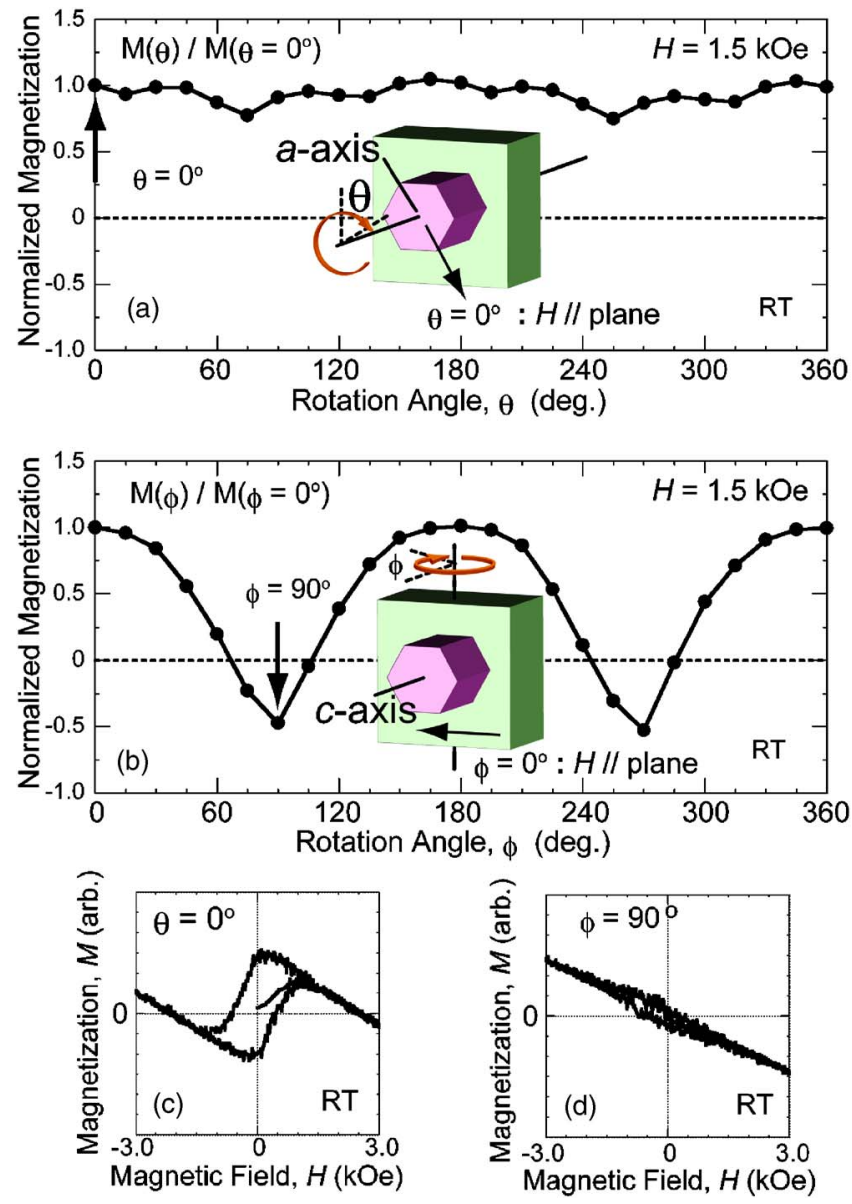

FIG. 3. (Color online) Magnetization dependences on the directions of applied $\mathbf{H}$ to the MnAs NCs on GaInAs (111)B surfaces: (a) in-plane and (b) out-of-plane rotations of the sample. $M-H$ curves observed at (c) $\theta=0^{\circ}$ in (a) and (d) $\phi=90^{\circ}$ in (b).

MBE-grown MnAs thin films have shown ferromagnetic coupling for $\mathbf{H}$ in a direction perpendicular to the GaAs (111)B wafer planes due to the lack of strong in-plane magnetic anisotropy possibly caused by poor crystalline quality. ${ }^{13}$ Therefore, the experimental results in the present work clearly indicate that well-oriented hexagonal MnAs NCs with respect to the wafer planes have been formed by MOVPE. For the MnAs NCs embedded in GaAs (Ref. 22) and GaInAs/InP (Ref. 23) (001) layers grown by MOVPE, ferromagnetic resonance studies have revealed that the $c$ axis of the NCs is parallel to a [-111] or [111] direction of ZBtype GaAs and GaInAs/InP layers. These facts also indicate that $\{111\}$ orientations of ZB-type structures are the preferred directions for hexagonal MnAs NCs because of the similarity of crystallographic structures. On the GaInAs (111)B surfaces, the size, density, and uniformity of MnAs NCs strongly depend on MOVPE conditions, such as $p\left[\left(\mathrm{CH}_{3} \mathrm{C}_{5} \mathrm{H}_{4}\right)_{2} \mathrm{Mn}\right]$, growth time, $\mathrm{V} / \mathrm{Mn}$ ratios, and $T_{g}$. Figure 4 shows the AFM images of MnAs NCs after optimizing MOVPE conditions. The $T_{g}$ and the $\mathrm{V} / \mathrm{Mn}$ ratio were $650{ }^{\circ} \mathrm{C}$ and 1125 , respectively. The hexagonal NCs measured $130-170 \mathrm{~nm}$ in diameter, and the averaged height was $47 \mathrm{~nm}$. The density of the NCs was estimated to be 6.1 $\times 10^{8} \mathrm{~cm}^{-2}$. Smaller NCs with higher uniformity and density have been grown presumably because the thermally activated surface diffusion of $\mathrm{Mn}$ adatoms decreases with decreasing $T_{g}$ and increasing a $\mathrm{V} / \mathrm{Mn}$ ratio on the basis of the kinetics.

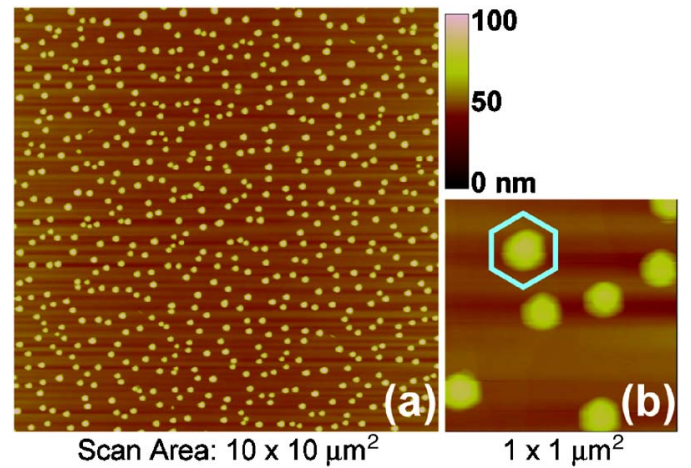

FIG. 4. (Color online) (a) Surface morphology of MnAs NC arrays selfassembled on GaInAs (111)B surfaces after optimizing MOVPE conditions and (b) highly magnified AFM image of the hexagonal NCs.

Detailed experimental results on growth condition dependence of the NC formation will be reported elsewhere. We believe that the hybridization of ferromagnetic MnAs NCs into 1D semiconductor NWs on (111)B layers is realized by the MOVPE techniques in the present work.

The authors thank J. Motohisa and A. Koike for fruitful discussions. This work was partly supported by the Grant-inAid for Scientific Research from the MEXT in Japan.

${ }^{1}$ M. Tanaka, J. P. Harbison, T. Sands, T. L. Cheeks, V. G. Keramidas, and G. M. Rothberg, J. Vac. Sci. Technol. B 12, 1091 (1994).

${ }^{2}$ H. Munekata, H. Ohno, S. von Molnar, A. Segmüller, L. L. Chang, and L. Esaki, Phys. Rev. Lett. 63, 1849 (1989).

${ }^{3}$ H. Ohno, A. Shen, F. Matsukura, A. Oiwa, A. Endo, S. Katsumoto, and Y. Iye, Appl. Phys. Lett. 69, 363 (1996)

${ }^{4}$ J. Shi, J. M. Kikkawa, D. D. Awschalom, G. Medeiros-Ribeiro, P. M. Petroff, and K. Babcock, J. Appl. Phys. 79, 5296 (1996).

${ }^{5}$ Th. Hartmann, M. Lampalzer, W. Stolz, K. Megges, J. Lorberth, P. J. Klar, and W. Heimbrodt, Thin Solid Films 364, 209 (2000).

${ }^{6}$ A. J. Blattner and B. W. Wessels, J. Vac. Sci. Technol. B 20, 1582 (2002).

${ }^{7}$ S. Hara, M. Lampalzer, T. Torunski, K. Volz, W. Treutmann, and W. Stolz,

J. Cryst. Growth 261, 330 (2004).

${ }^{8}$ S. Hara and A. Kuramata, Nanotechnology 16, 957 (2005).

${ }^{9}$ T. Slupinski, H. Munekata, and A. Oiwa, Appl. Phys. Lett. 80, 1592 (2002).

${ }^{10}$ S. Ohya, H. Shimizu, Y. Higo, J. Sun, and M. Tanaka, Jpn. J. Appl. Phys., Part 2 41, L24 (2002).

${ }^{11}$ H. Shimizu and M. Tanaka, Appl. Phys. Lett. 81, 5246 (2002).

${ }^{12}$ M. Ramsteiner, H. Y. Hao, A. Kawaharazuka, H. J. Zhu, M. Kästner, R. Hey, L. Däweritz, H. T. Grahn, and K. H. Ploog, Phys. Rev. B 66, 081304(R) (2002).

${ }^{13}$ M. Tanaka, K. Saito, and T. Nishinaga, Appl. Phys. Lett. 74, 64 (1999).

${ }^{14}$ Y. Morishita, K. Iida, J. Abe, and K. Sato, Jpn. J. Appl. Phys., Part 2 36, L1100 (1997).

${ }^{15}$ J. Sadowski, J. Kanski, L. Ilver, and J. Johansson, Appl. Surf. Sci. 166, 247 (2000).

${ }^{16}$ S. Sugahara and M. Tanaka, J. Appl. Phys. 89, 6677 (2001).

${ }^{17}$ M. Kästner, L. Däweritz, and K. H. Ploog, Surf. Sci. 511, 323 (2002).

${ }^{18}$ N. Mattoso, M. Eddrief, J. Varalda, A. Ouerghi, D. Demaille, V. H. Etgens, and Y. Garreau, Phys. Rev. B 70, 115324 (2004).

${ }^{19}$ A. G. Banshchikov, A. V. Kimel', R. V. Pisarev, A. A. Rzhevskiŭ, N. S. Sokolov, A. Keen, Th. Rasing, Ahsan M. Nazmul, and M. Tanaka, Phys. Solid State 43, 1941 (2002).

${ }^{20}$ J. Motohisa, J. Noborisaka, J. Takeda, M. Inari, and T. Fukui, J. Cryst. Growth 272, 180 (2004).

${ }^{21}$ J. Noborisaka, J. Motohisa, S. Hara, and T. Fukui, Appl. Phys. Lett. 87, 093109 (2005).

${ }^{22}$ Th. Hartmann, M. Lampalzer, P. J. Klar, W. Stolz, W. Heimbrodt, and H.-A. Krug von Nidda, A. Loidl, and L. Svistov, Physica E (Amsterdam) 13, 572 (2002).

${ }^{23}$ M. Leuschner, P. J. Klar, W. Heimbrodt, W. W. Rühle, S. Hara, W. Stolz, K. Volz, T. Kurz, A. Loidl, and H.-A. Krug von Nidda, J. Magn. Magn. Mater. 301, 478 (2006). 